\title{
Fatores ambientais e risco de quedas em idosos: revisão sistemática
}

\author{
Environmental hazards and risk of fall in the elderly: systematic review
}

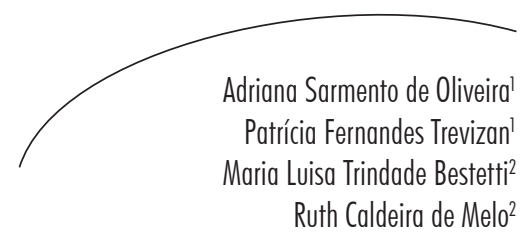

\section{Resumo}

A queda em idosos é resultado de uma interação complexa entre fatores intrínsecos e extrínsecos. Embora seja difícil separar esses fatores, estudos apontam que fatores de risco ambientais estão presentes em aproximadamente $40 \%$ das quedas. O objetivo deste estudo foi realizar uma revisão sistemática da literatura sobre o envolvimento de fatores ambientais nas quedas em idosos vivendo na comunidade. Para tanto, foram selecionados estudos publicados no período de janeiro de 2000 a maio de 2014 nas bases de dados eletrônicas MEDLINE, LILACS e SciELO. Apenas artigos disponíveis na íntegra e em inglês, português e espanhol foram considerados para esta revisão. Após a análise do título, do resumo e do texto na íntegra, dez artigos foram incluídos na revisão. Nos estudos analisados, aproximadamente metade das quedas ocorreu durante a locomoção e envolveu tropeços e escorregões. Os fatores de risco ambientais estão muito presentes nas quedas (20-58\%), sendo que superfícies irregulares, superfícies molhadas/escorregadias, objetos/tapetes soltos e desníveis no chão/problemas com degraus foram os mais prevalentes. Observou-se tendência de aumento na ocorrência de quedas em ambientes externos, as quais são frequentemente precipitadas por fatores extrínsecos. Mais estudos são necessários na caracterização e no desenvolvimento de estratégias de prevenção de quedas em ambientes externos.

\section{Abstract}

Falls in the elderly is the result of a complex interplay between intrinsic and extrinsic factors. Although it is difficult to separate these factors, studies indicate that environmental hazards are involved in approximately $40 \%$ of the falls. This study aimed to conduct a systematic review about the contribution of environmental hazards for falls in community-dwelling elderly. Studies published from January 2000 to May 2014 in the electronic databases MEDLINE, LILACS and SciELO were selected. Only free full-text articles written in English, Portuguese and Spanish were considered

\footnotetext{
Unidade de Reabilitação Cardiovascular e Fisiologia do Exercício, Instituto do Coração, Hospital das Clínicas, Faculdade de Medicina. Universidade de São Paulo. São Paulo, SP, Brasil.

2 Curso de Gerontologia, Escola de Artes, Ciências e Humanidades. Universidade de São Paulo. São Paulo, SP, Brasil.
}

Palavras-chave: Acidentes por Quedas. Idoso. Medicina Ambiental. 
for this research. After title, abstract and full text analysis, ten articles were included in this review. In the studies analyzed, approximately half of the falls occurred during walk and involved tripping and slipping. The environmental risk factors are present in falls (20-58\%), in which irregular surfaces, wet/slippery floors, objects/loose rugs and uneven floor/steps were the most prevalent hazards among the studies. There was tendency of increase in the occurrence of outdoor falls, which are often caused by extrinsic factors. More studies are needed to characterize and develop strategies to prevent outdoor falls among community-dwelling older adults.
Key words: Accidental Falls. Elderly. Environmental Medicine.

\section{INTRODUÇÃO}

A queda pode ser definida como uma mudança inesperada e não intencional de posição, que leva inadvertidamente o indivíduo a um nível inferior. ${ }^{1}$ Devido a sua repercussão na saúde dos idosos, a queda é considerada um evento limite, pois em geral está associada a fragilidade, dependência, institucionalização e morte. ${ }^{2}$ Sendo assim, as quedas são consideradas um problema de saúde pública, já que sua ocorrência está relacionada a altas taxas de morbimortalidade, além do elevado custo social e econômico. ${ }^{3}$

Nos Estados Unidos, acidentes e lesões não intencionais ocupam o quinto lugar entre as causas de morte na população idosa, sendo as quedas responsáveis por dois terços destas. ${ }^{4}$ Dentre os idosos americanos que vivem na comunidade, cerca de $28-40 \%$ cairão pelo menos uma vez, no período de um ano. ${ }^{1,4}$ A ocorrência de quedas no Brasil não é muito diferente dos padrões observados em outros países. Aproximadamente $30-40 \%$ dos idosos brasileiros, residentes na comunidade, caem ao menos uma vez por ano, enquanto $11 \%$ caem de forma recorrente. ${ }^{5,6}$

É importante destacar ainda que a incidência de quedas aumenta expressivamente a partir dos 75 anos de idade, sendo que as mulheres apresentam maior risco de cair que os homens., ${ }^{4,5}$ Essa diferença pode ser explicada pela maior fragilidade, prevalência de doenças crônicas, consumo de medicações e longevidade das mulheres em relação aos homens. ${ }^{5,7}$
Embora a maioria das quedas não resulte em lesões graves, aproximadamente $5 \%$ das quedas em idosos residentes na comunidade resultam em fraturas ou hospitalização. ${ }^{4}$ Entretanto, muitos idosos relatam episódios de queda apenas quando a mesma lhes traz sérias consequências, ignorando as quedas que não lhes provocaram lesões, por acreditarem que estas são inerentes ao envelhecimento.

A fratura é a consequência mais frequentemente observada nos casos que necessitam de hospitalização, seguida do medo de cair e necessidade de auxílio para atividades de vida diária. ${ }^{9-11}$ Sendo assim, além das lesões físicas, a queda pode trazer consequências psicológicas. $\mathrm{O}$ medo de sofrer uma nova queda faz com que os idosos restrinjam suas atividades diárias, contribuindo assim para o aumento da inatividade e o declínio da capacidade funcional., ${ }^{411,12}$

Devido às características multifatoriais das quedas, estabelecer uma única causa é muitas vezes impossível. Além disso, a maioria das quedas é resultado de uma interação complexa entre diferentes fatores de risco, ${ }^{13}$ os quais podem ser classificados, de acordo com sua natureza, em intrínsecos e extrínsecos. ${ }^{11,14}$

Os fatores intrínsecos são aqueles relacionados ao próprio idoso e refletem a incapacidade, pelo menos parcial, de o mesmo manter ou recuperar o equilíbrio quando houver um deslocamento acentuado do centro de gravidade. Alterações fisiológicas relacionadas ao envelhecimento, 
presença de doenças agudas, fraqueza muscular e alterações da marcha, por exemplo, podem prejudicar a capacidade de manter o equilíbrio.,11

Os fatores extrínsecos, por sua vez, estão associados ao ambiente físico no qual o idoso se encontra (piso escorregadio, tapetes soltos, objetos em áreas de circulação, ausência de barras de apoio e corrimãos, móveis instáveis e iluminação inadequada). ${ }^{11}$ Fatores de risco ambientais também são determinantes para as quedas e não menos importantes que os demais, já que estes estão presentes em aproximadamente $30-50 \%$ das quedas. ${ }^{4}$

Considerando a repercussão das quedas na saúde dos idosos, bem como nos gastos despendidos no tratamento de suas consequências, se faz importante identificar fatores de risco para as quedas e desenvolver intervenções eficazes para o adequado planejamento de ações preventivas, sejam essas de caráter primário ou secundário. Nesse sentido, o presente estudo teve como objetivo realizar uma revisão sistemática da literatura do papel do ambiente nas quedas por meio da seleção e análise de artigos científicos que investigaram a presença de fatores de risco extrínsecos relacionados a quedas em idosos vivendo em comunidade.

\section{MATERIAIS E MÉTODOS}

\section{Estratégia de pesquisa}

A pesquisa dos artigos foi realizada nas bases de dados eletrônicas MEDLINE ((Medical Literature Analysis and Retrieval System Online)), LILACS (Literatura Latino-americana e do Caribe em Ciências da Saúde) e SciELO (Scientific Electronic Library Online).

Os descritores utilizados na busca foram: idoso, quedas, risco extrínseco, risco ambiental, e seus correspondentes em inglês (older, elderly, aged, falls, extrinsic factors, environmental risk, environmental hazard) e em espanhol (anciano, caídas, riesgo ambiental, riesgo extrínseco).
Apenas artigos em inglês, português e espanhol foram considerados para esta revisão. Além do idioma de publicação, foram considerados como limite na estratégia de busca os textos disponíveis na íntegra e a presença dos descritores no título ou resumo.

\section{Seleção dos artigos}

Foram selecionados para esta revisão estudos publicados no período de janeiro de 2000 a maio de 2014. Em relação aos participantes, a inclusão dos artigos foi limitada aos estudos com idosos (maiores que 60 anos), vivendo na comunidade. $\mathrm{Na}$ seleção, foram considerados os artigos que incluíssem os fatores de risco extrínsecos entre os determinantes para as quedas na sua descrição. Os artigos que obedeceram aos critérios de inclusão foram analisados de forma independente por dois revisores.

\section{Análise dos artigos}

A busca foi realizada por dois revisores, que avaliaram os títulos e resumos de forma independente; caso houvesse alguma discordância entre eles quanto à seleção dos artigos, esta seria resolvida por um terceiro revisor. Após a primeira seleção, os artigos foram lidos na íntegra e aqueles que não estivessem dentro dos critérios estabelecidos foram excluídos do estudo.

\section{Descrição dos artigos}

Com o intuito de auxiliar na visualização dos principais resultados dos artigos selecionados durante a busca nas bases de dados, informações referentes aos autores, às características dos participantes, à prevalência de quedas, à circunstância das quedas e aos fatores de risco extrínsecos estão apresentadas no quadro 1. 


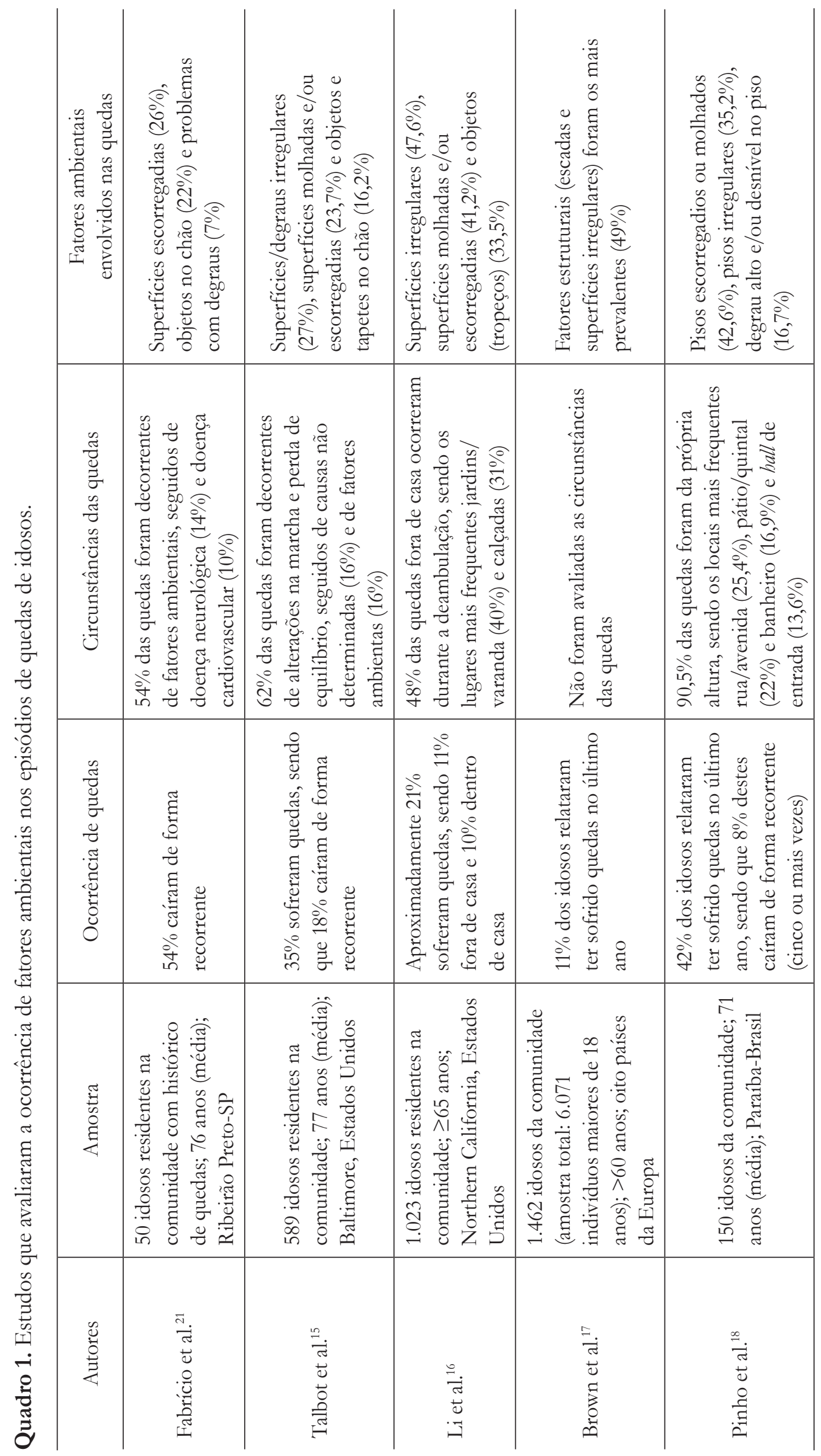




\begin{tabular}{|c|c|c|c|c|c|}
\hline 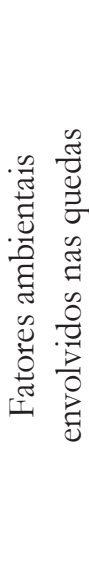 & 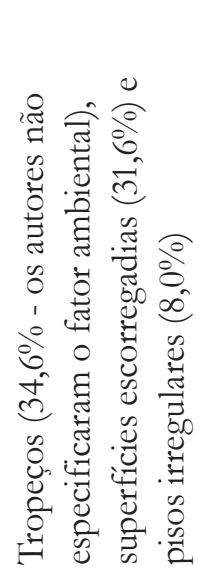 & 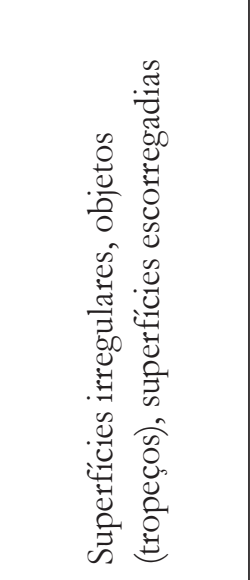 & 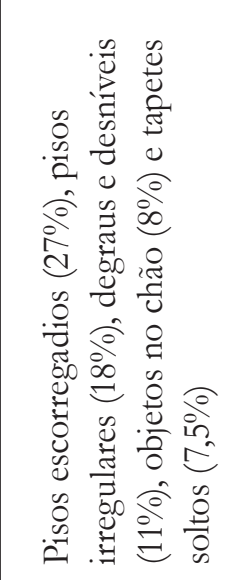 & 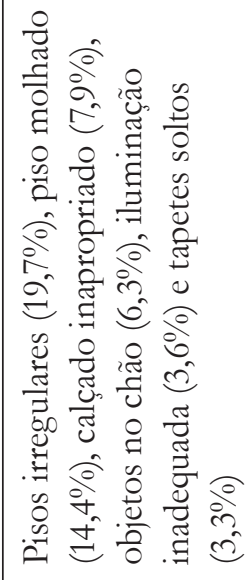 & 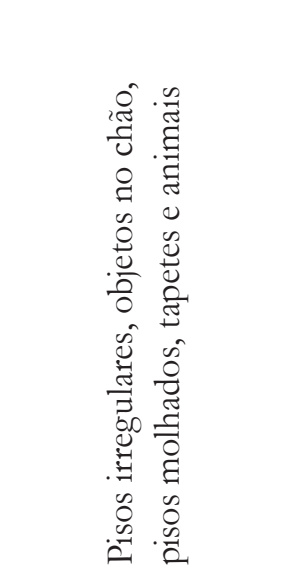 \\
\hline 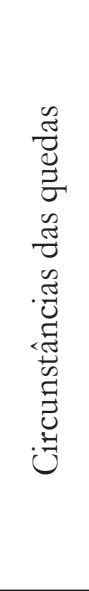 & 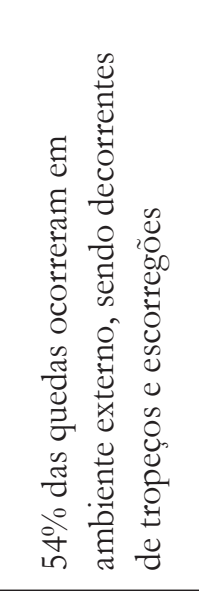 & 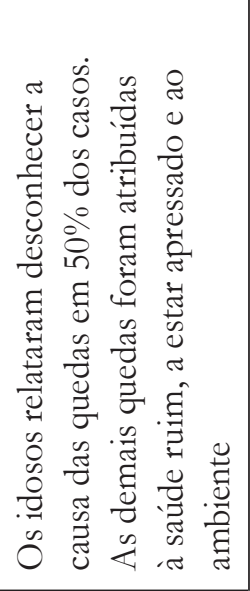 & 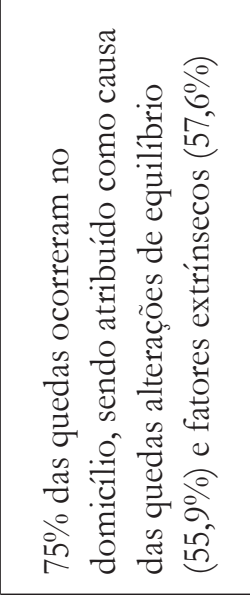 & 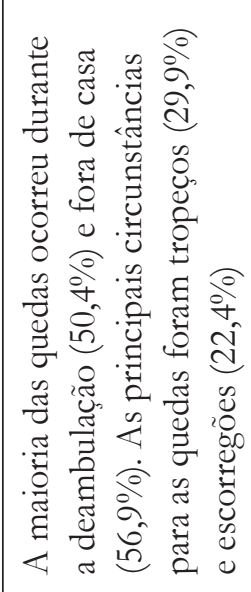 & 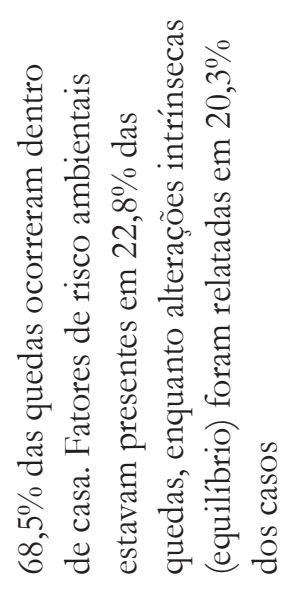 \\
\hline 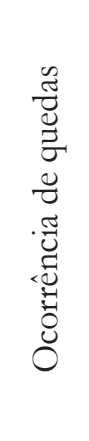 & 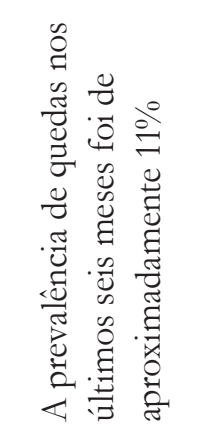 & 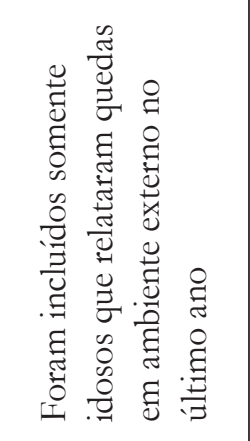 & 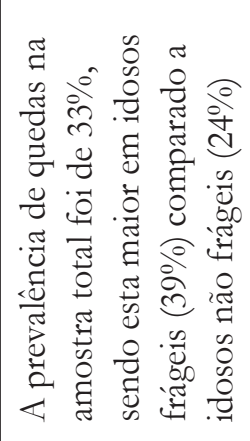 & 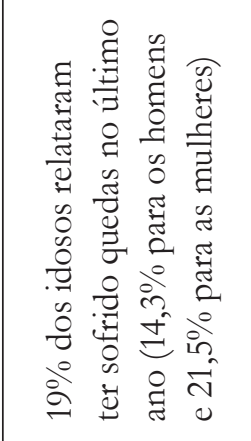 & 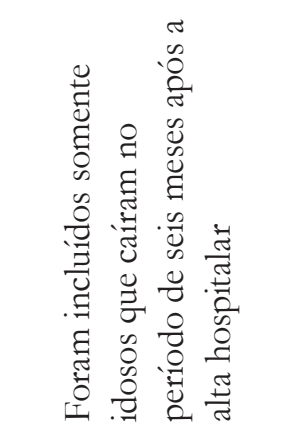 \\
\hline & 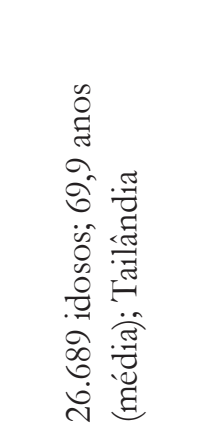 & 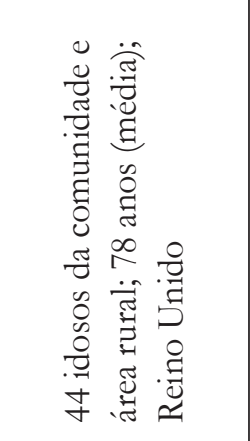 & 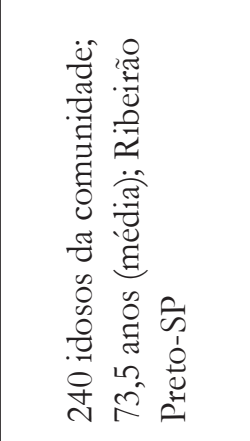 & 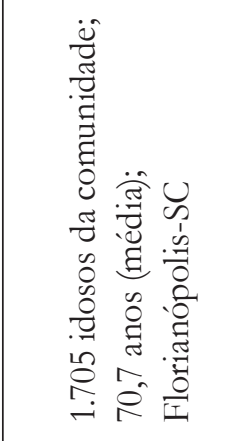 & 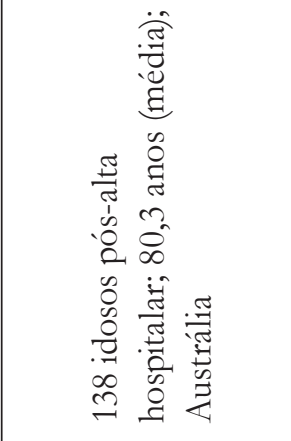 \\
\hline$\frac{1}{4}$ & 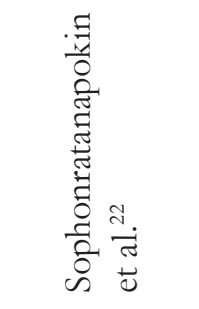 & 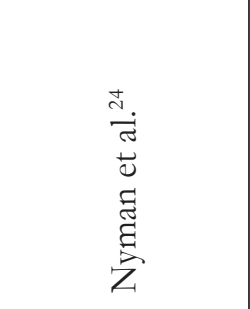 & $\begin{array}{l}\bar{U} \\
\tilde{E} \\
\vec{I}\end{array}$ & 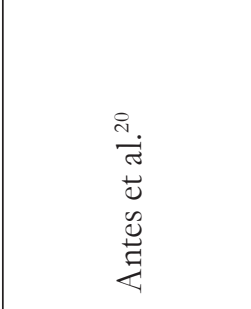 & $\stackrel{\breve{\Xi}}{\bar{\Xi}}$ \\
\hline
\end{tabular}




\section{RESULTADOS}

\section{Estudos selecionados}

$\mathrm{Na}$ busca inicial utilizando os descritores relacionados aos fatores de risco extrínsecos, foram identificados nas bases de dados eletrônicas MEDLINE, SciELO e LILACS, respectivamente 203, 134 e 18 artigos. Após análise do título, foram excluídos 156 do MEDLINE, 116 do SciELO e 12 do LILACS, por não abordarem o tema da pesquisa, incluírem idosos institucionalizados ou hospitalizados e, ainda, por incluírem idosos com doenças e alterações específicas (por exemplo, doença de Parkinson).

Após a leitura dos resumos foram selecionados, especificamente sobre fatores de risco extrínsecos e quedas, oito artigos do MEDLINE, cinco do SciELO e dois do LILACS. Na análise final dos artigos, cinco foram excluídos por avaliarem fatores extrínsecos que não estavam diretamente relacionados com as quedas da amostra estudada.

\section{Características dos estudos selecionados}

Nos estudos analisados, a prevalência de quedas no último ano em idosos da comunidade variou entre 11 e $42 \%,{ }^{15-20}$ sendo observada menor prevalência $(11 \%$ a $21 \%)$ nos estudos de base populacional. ${ }^{16,17,20}$ Dos dez artigos incluídos no presente estudo, sete avaliaram a ocorrência de quedas tanto em ambientes interno como externo, ${ }^{15,16,18-23}$ enquanto um analisou as quedas em ambiente externo ${ }^{24}$ e um dentro do domicílio. ${ }^{17}$ Em relação às circunstâncias das quedas, aproximadamente a metade (48-56,5\%) ocorreu durante a deambulação ${ }^{15,16,20}$ e envolveu principalmente tropeços e escorregões (22,4$34,6 \%) \cdot{ }^{16,20,22}$

Nos estudos que avaliaram as causas das quedas, os fatores de risco ambientais estiveram presentes em $16 \%$ a $58 \% \%^{15,19,21,23}$ dos eventos, enquanto $20 \%$ a $62 \%$ das quedas foram atribuídas a alterações da marcha e equilíbrio..$^{15,19,23}$ Os fatores de risco extrínsecos relacionados às quedas incluíam superfícies irregulares (18 a
$48 \%)^{16,18-20}$ e superfícies molhadas/escorregadias (14 a 43\%), ${ }^{15,16,19-21}$ seguidos por objetos/tapetes soltos (10 a 33,5\%), ${ }^{15,16,19-21}$ desníveis no chão/ problemas com degraus ( 7 a $17 \%$ ), ${ }^{18-21}$ problemas com calçados $(6,3 \%)^{20}$ e iluminação inadequada $(3,6 \%){ }^{20}$

Ao comparar a prevalência de quedas dentro e fora do domicílio, foi possível observar que mais da metade (55\%) das quedas ocorreu em ambiente externo ao domicílio, principalmente nos estudos de base populacional. ${ }^{16,20,22}$ Por outro lado, quando o estudo incluía idosos frágeis ou com problemas de saúde na sua amostra, a prevalência de quedas no domicílio foi maior $(66 \%$ a $75 \%){ }^{19,21,23}$ Os locais externos de maior ocorrência de quedas são jardins, pátios, calçadas e ruas. ${ }^{16,20}$

\section{DISCUSSÃO}

Em idosos residentes na comunidade, fatores de risco ambientais se mostram presentes em grande porcentagem das quedas, ${ }^{15,19,21,23}$ independentemente do local de ocorrência, ou seja, tanto fora como dentro de casa. Em estudo envolvendo 26.689 idosos, Soponratanapolin et al. ${ }^{22}$ mostraram que fatores de risco extrínsecos também podem ser importantes preditores de quedas em idosos, uma vez que a chance de cair é $1,39 \%$ maior naqueles que residem em casas com pisos escorregadios.

Em relação às circunstâncias das quedas, a atividade mais prevalente no momento da queda é a deambulação, ${ }^{15,16,20}$ sendo escorregões e tropeços os principais fatores precipitantes. ${ }^{20,22}$ Outras atividades relatadas durante as quedas incluem atividades domésticas, descer escadas, banho e transferências. ${ }^{15,20}$ Em geral, tropeços ocorrem durante a fase de balanço da marcha, quando o pé encontra um obstáculo durante a sua movimentação para frente..$^{14,25}$

A incapacidade de evitar uma queda, principalmente devido a um tropeço, pode estar associada às alterações próprias do envelhecimento (alterações na marcha, déficit visual, redução de força muscular, redução no tempo de reação e 
déficit cognitivo). ${ }^{26}$ Em relação às alterações no padrão de marcha, idosos apresentam redução do comprimento e da altura das passadas, redução da velocidade da marcha e aumento da base de apoio. ${ }^{27}$ Embora essas alterações proporcionem maior estabilidade durante a deambulação, a redução na altura das passadas, por exemplo, pode predispor o idoso a tropeços. ${ }^{27}$

Segundo Lord et al., ${ }^{28}$ a redução da acuidade visual associada à idade também é importante fator de risco para quedas em idosos, já que obstáculos podem não ser percebidos a tempo de evitar a queda. Esses autores sugerem, ainda, que a percepção de profundidade e a sensibilidade para identificar contrastes influenciam na capacidade de manter o equilíbrio e evitar possíveis riscos ambientais. Como o envelhecimento é acompanhado de redução da força muscular e aumento do tempo de reação, a habilidade de reagir e/ou recuperar o equilíbrio frente a um risco ambiental encontra-se reduzida no idoso, comparativamente a indivíduos jovens, sobretudo quando o obstáculo é percebido pouco antes de ser atingido. ${ }^{26,27}$

Os fatores de risco ambientais mais prevalentes nos estudos avaliados incluem superfícies irregulares, pisos escorregadios/molhados e objetos/tapetes nas áreas de circulação. 15,16,18-22 Em relação ao lugar de ocorrência das quedas, os estudos revisados apresentaram resultados divergentes. Nos estudos envolvendo um número menor de idosos, a prevalência de quedas dentro do domicílio (75\%) foi maior que as que ocorreram em ambiente externo $(25 \%))^{18,19,23}$ principalmente se os idosos apresentam algum problema de saúde. ${ }^{19,23}$ Hill et al., ${ }^{23}$ por exemplo, avaliaram a incidência de quedas em idosos nos seis meses seguintes à alta hospitalar. No período de seguimento desse estudo, $68,5 \%$ das quedas ocorreram dentro de casa, sendo os locais mais frequentes o quarto $(29 \%)$, a sala $(19,6 \%)$ e o banheiro $(14,5 \%)$. Os autores observaram também que indivíduos que necessitam de auxílio nas atividades de vida diária apresentam mais chances de cair dentro de casa do que no ambiente externo.
Por outro lado, em três estudos de base populacional, ${ }^{16,20,22}$ mais da metade das quedas $(54-56,9 \%)$ ocorreu fora de casa, mais especificamente, em calçadas, meios-fios e ruas. ${ }^{16,20}$ Outros locais externos de ocorrência de quedas incluem jardins, varandas/sacadas, parques, garagens e estacionamentos. ${ }^{16,20}$ Ao avaliarem qualitativamente as quedas em ambientes externos, Nyman et al. ${ }^{24}$ observaram que estas ocorrem frequentemente em áreas familiares ao idoso, muitas vezes próximas ao domicílio, sendo que o local da queda pode estar associado aos aspectos da saúde dos mesmos. Segundo Nyman et al., ${ }^{24}$ idosos mais ativos fisicamente, com boas condições de saúde e boa capacidade funcional são mais propensos a cair em ambientes externos e a considerar os fatores ambientais como principais precipitadores das quedas. Li et al. ${ }^{16}$ avaliaram as características das quedas que ocorrem fora de casa e observaram que alto nível de atividade física de lazer é um preditor independente para quedas em ambientes externos, enquanto a presença de problemas de saúde e limitações físicas são preditores independentes para quedas dentro do domicílio.

Considerando os estudos discutidos anteriormente, o risco ambiental isolado parece não ser suficiente para causar uma queda, mas sim a interação entre as habilidades dos idosos e o grau de exposição ao fator de risco ambiental. ${ }^{29}$ Por isso, idosos com boa percepção de saúde e com histórico de quedas fora de casa são mais propensos a atribuir suas quedas a fatores ambientais, enquanto aqueles com percepção de saúde ruim e com dificuldades de movimento atribuem suas quedas às próprias limitações. ${ }^{30}$ É possível, então, que idosos com capacidade funcional preservada se exponham a ambientes de alta demanda, enquanto idosos frágeis sejam capazes de lidar apenas com ambientes de baixa demanda, o que explicaria parte das diferenças entre os idosos que caem dentro e fora de casa. ${ }^{29,30}$

A temática das quedas tem sido foco de diversos estudos nos últimos anos; entretanto, ainda existem poucas informações sobre as quedas que ocorrem em ambiente externo. Na presente 
revisão, estudos recentes envolvendo amostras representativas da população ${ }^{16,20,22}$ mostraram uma tendência de mudança na epidemiologia das quedas, caracterizada sobretudo por aumento da ocorrência de quedas em ambientes externos.

Com o aumento da expectativa de vida e o surgimento de políticas públicas com foco no envelhecimento ativo, ${ }^{31,32}$ é esperado um crescimento na parcela dos idosos com melhores condições de saúde vivendo e contribuindo ativamente dentro da comunidade. Nesse cenário, as quedas em ambientes externos, na maioria das vezes precipitadas por fatores ambientais, ${ }^{24}$ passam a ser um grande desafio, tanto no que diz respeito à identificação de idosos em risco como no planejamento de estratégias preventivas.

\section{CONCLUSÃO}

Com base nos estudos revisados, pode-se concluir que a prevalência de quedas em idosos da comunidade varia entre 10 e $40 \%$, havendo

\section{REFERÊNCIAS}

1. Masud T, Morris RO. Epidemiology of falls. Age Ageing 2001;30(Suppl 4):3-7.

2. Ensrud KE, Ewing SK, Taylor BC, Fink HA, Stone KL, Cauley JA, et al. Frailty and risk of falls, fracture, and mortality in older women: the study of osteoporotic fractures. J Gerontol Ser A Biol Sci Med Sci 2007;62(7):744-51.

3. Gelbard R, Inaba K, Okoye OT, Morrell M, Saadi Z, Lam L, et al. Falls in the elderly: a modern look at an old problem. Am J Surg 2014;208(2):249-53.

4. Rubenstein LZ. Falls in older people: epidemiology, risk factors and strategies for prevention. Age Ageing 2006;35(Suppl 2):ii37-41.

5. Perracini MR, Ramos LR. Fatores associados a quedas em uma coorte de idosos residentes na comunidade. Rev Saúde Pública 2002;36(6):709-16.

6. Siqueira FV, Facchini LA, Piccini RX, Tomasi E, Thumé E, Silveira DS, et al. Prevalência de quedas em idosos e fatores associados. Rev Saúde Pública. 2007;41(5):749-56. tendência de menor taxa de ocorrência nos estudos de base populacional. Além disso, aproximadamente metade das quedas ocorre durante a locomoção e envolve tropeços e escorregões.

Em geral, fatores de risco ambientais estão presentes em grande parte das quedas (20-58\%), sendo que superfícies irregulares, superfícies molhadas/escorregadias, objetos/tapetes soltos e desníveis no chão/problemas com degraus foram os mais prevalentes nos estudos analisados. O local de ocorrência das quedas parece sofrer influência de fatores intrínsecos, uma vez que idosos ativos fisicamente tendem a cair em ambientes externos, enquanto idosos com comprometimento funcional caem dentro do próprio domicílio.

Com o aumento da expectativa de vida e da participação dos idosos na comunidade, mais estudos devem focar na caracterização das quedas em ambientes externos, bem como no desenvolvimento de estratégias de prevenção.

7. Saari P, Heikkinen E, Sakari-Rantala R, Rantanen T. Fall-related injuries among initially 75 - and 80 -year old people during a 10-year follow-up. Arch Gerontol Geriatr 2007;45(2):207-15.

8. Zecevic AA, Salmoni AW, Speechley M, Vandervoort AA. Defining a fall and reasons for falling: comparisons among the views of seniors, health care providers, and the research literature. Gerontologist 2006;46(3):367-76.

9. Friedman SM, Munoz B, West SK, Rubin GS, Fried LP. Falls and fear of falling: which comes first? A longitudinal prediction model suggests strategies for primary and secondary prevention. J Am Geriatr Soc 2002;50(8):1329-35.

10. Hartholt KA, Stevens JA, Polinder S, Van Der Cammen TJM, Patka P. Increase in fall-related hospitalizations in the United States, 2001-2008. J Trauma 2011;71(1):255-8.

11. Ambrose AF, Paul G, Hausdorff JM. Risk factors for falls among older adults: a review of the literature. Maturitas 2013;75(1):51-61. 
12. Zijlstra GAR, Van Haastregt JCM, Van Eijk JTM, Van Rossum E, Stalenhoef PA, Kempen GIJM. Prevalence and correlates of fear of falling, and associated avoidance of activity in the general population of community-living older people. Age Ageing 2007;36(3):304-9.

13. Voermans NC, Snijders AH, Schoon Y, Bloem BR. Why old people fall (and how to stop them). Pract Neurol 2007;7(3):158-71.

14. Bueno-Cavanillas A, Padilla-Ruiz F, Jiménez-Moleón JJ, Peinado-Alonso CA, Gálvez-Vargas R. Risk factors in falls among the elderly according to extrinsic and intrinsic precipitating causes. Eur J Epidemiol 2000;16(9):849-59.

15. Talbot LA, Musiol RJ, Witham EK, Metter EJ. Falls in young, middle-aged and older community dwelling adults: perceived cause, environmental factors and injury. BMC Public Health 2005;5:86.

16. Li W, Keegan THM, Sternfeld B, Sidney S, Quesenberry CP Jr, Kelsey JL. Outdoor Falls Among Middle-Aged and Older Adults: A Neglected Public Health Problem. Am J Public Health 2006;96(7):1192-200.

17. Brown MJ, Jacobs DE. Residential light and risk for depression and falls: results from the LARES study of eight European cities. Public Health Rep 2011;126 (Suppl 1):131-40.

18. Pinho TAM, Silva AO, Tura LFR, Moreira MASP, Gurgel SN, De Azevedo Freitas Smith A, et al. Avaliação do risco de quedas em idosos atendidos em Unidade Básica de Saúde. Rev Esc Enferm USP 2012;46(2):320-7.

19. Fhon JRS, Rosset I, Freitas CP, Silva AO, Santos JLF, Rodrigues RAP. Prevalencia de quedas de idosos em situação de fragilidade. Rev Saúde Pública 2013;47(2):266-73.

20. Antes DL, D’Orsi E, Benedetti TRB. Circumstances and consequences of falls among the older adults in Florianopolis. Epi Floripa Aging 2009. Rev Bras Epidemiol 2013;16(2):469-81.

21. Fabrício SCC, Rodrigues RAP, Costa Junior MLD. Causas e conseqüências de quedas de idosos atendidos em hospital público. Rev Saúde Pública 2004;38(1):93-9.

22. Sophonratanapokin B, Sawangdee Y, Soonthorndhada $K$. Effect of the living environment on falls among the elderly in Thailand. Southeast Asian J Trop Med Public Healt 2012;43(6):1537-47.

23. Hill AM, Hoffmann T, Haines TP. Circumstances of falls and falls-related injuries in a cohort of older patients following hospital discharge. Clin Interv Aging 2013;8:765-74.

24. Nyman SR, Ballinger C, Phillips JE, Newton R. Characteristics of outdoor falls among older people: a qualitative study. BMC Geriatr 2013;13:1-14.

25. Berg WP, Alessio HM, Mills EM, Tong C. Circumstances and consequences of falls in independent community-dwelling older adults. Age Ageing 1997;26(4):261-8.

26. Van Dieën JH, Pijnappels M, Bobbert MF. Age-related intrinsic limitations in preventing a trip and regaining balance after a trip. Saf Sci 2005;43(7):437-53.

27. Pijnappels M, Reeves ND, Maganaris CN, Van Dieën JH. Tripping without falling; lower limb strength, a limitation for balance recovery and a target for training in the elderly. J Electromyogr Kinesiol 2008;18(2):188-96.

28. Lord SR, Dayhew J. Visual risk factors for falls in older people. J Am Geriatr Soc 2001;49(5):508-15.

29. Lord SR, Menz HB, Sherrington C. Home environment risk factors for falls in older people and the efficacy of home modifications. Age Ageing 2006;35(Suppl 2):ii55-9.

30. Weinberg LE, Strain LA. Community-dwelling older adults' attributions about falls. Arch Phys Med Rehabil 1995;76(10):955-60.

31. Boudiny K. "Active ageing": from empty rhetoric to effective policy tool. Ageing Soc 2013;33(6):1077-98.

32. Fuchs J, Scheidt-Nave C, Hinrichs T, Mergenthaler A, Stein J, Riedel-Heller SG, et al. Indicators for healthy ageing: a debate. Int J Environ Res Public Health. 2013;10(12):6630-44. 